Relations industrielles

Industrial Relations

\title{
Lallement, Michel, Les gouvernances de l'emploi: relations professionnelles et marché du travail en France et en Allemagne
}

\section{Gilles L. Bourque}

Volume 55, numéro 1, 2000

URI : https://id.erudit.org/iderudit/051300ar

DOI : https://doi.org/10.7202/051300ar

Aller au sommaire du numéro

Éditeur(s)

Département des relations industrielles de l'Université Laval

ISSN

0034-379X (imprimé)

1703-8138 (numérique)

Découvrir la revue

Citer ce compte rendu

Bourque, G. L. (2000). Compte rendu de [Lallement, Michel, Les gouvernances de l'emploi: relations professionnelles et marché du travail en France et en Allemagne]. Relations industrielles / Industrial Relations, 55(1), 180-182. https://doi.org/10.7202/051300ar

Tous droits réservés (C Département des relations industrielles de l'Université Laval, 2000
Ce document est protégé par la loi sur le droit d'auteur. L'utilisation des services d’Érudit (y compris la reproduction) est assujettie à sa politique d'utilisation que vous pouvez consulter en ligne.

https://apropos.erudit.org/fr/usagers/politique-dutilisation/ 
adopt, the original implicit value set. Because all team members knew the rules and had accepted their importance in shaping behaviours that produced the outcomes the team (and the organization) valued, team members were also expected to enforce the rules on one another. Thus, Barker documents two kinds of pressure on team members : not only to comply with the rules themselves, but also to ensure that the rules are complied with by the rest of the team's members.

The system Barker describes creates stress for those working in it. Team members describe for him the tension they feel working in this system with its accompanying, omnipresent pressure to follow the "rules" and to enforce them on others. Those who comply are rewarded by being made to feel an integral part of the team; while those who are perceived as failing to display the correct behaviours and attitudes are punished with guilt, peer pressure, and ultimately the loss of their job. Barker argues that the team system of value identification, adoption, and on-going monitoring creates a powerful system of supervision - much more controlling, he maintains, than the traditional supervisory hierarchy. Rather than Weber's iron cage, Barker points out that selfmanaged team members now live in a "lacy, steel-filigree cage" of their own making where, although team members feel more in control, they are certainly more controlled. Yet, much of this control takes place unbeknownst to workers. Concertive control, Barker states, is for team members, "a natural, seamless, and exceedingly subtle part of their lives" (note, p. 165).
The strength of Barker's work lies in the documenting of the process by which a team's system of concertive control is constructed. His insightful, indepth case evidence is compelling and the theoretical development he makes is a singular contribution of this work. Disappointingly, however, Barker fails to adequately address the negative consequences of the system he so carefully describes. Although explicitly setting out to understand the negative consequences of the system of concertive control so that it could be made better for both workers and the organization, Barker, in the end, sidesteps the issue. $\mathrm{He}$ in fact produces no evidence that the system leads to anything but ideal outcomes from the organization's perspective. Thus, he is confronted with the possibility that workers' can be made better off (he does document negative consequences for workers of the system) only by making the organization worse off. By working from a unitary perspective, in which he has assumed workers' and the organization's interests are aligned, Barker finds himself unable to address how the system can be made better for workers. Recognizing the potential for conflicting interests in the employment relationship would have enabled Barker to address the negative impacts of such a system on workers in a more substantive way and would have added considerably to the value of this book. Nonetheless, Barker's work makes a significant contribution to the field and students, unions, and industrial relations scholars would all gain valuable insights from this work.

ANN C. Frost

University of Western Ontario

\section{Les gouvernances de l'emploi : relations professionnelles et marché du travail en France et en Allemagne par Michel LALLEMENT, Paris : Desclée de Brouwer, 1999, 252 p., ISBN 2 - 220-04443-2.}

Comme l'indique le sous-titre, le plus récent ouvrage de Michel Lallement porte sur les relations professionnelles et le marché du travail en France et en 
Allemagne. L'auteur connaît bien, pour les avoir étudiés depuis de nombreuses années, tant les deux thèmes que les deux pays abordés dans cet ouvrage. Les études qu'il leur a consacrées ont mené à des monographies d'entreprises dans les deux pays ou à des rapports de recherche portant plus spécifiquement sur le thème de la flexibilisation du travail et sur les conséquences de la réunification allemande sur les relations professionnelles de ce pays.

C'est en mobilisant ce matériau empirique, produit au cours de la décennie, que l'auteur désire contribuer au mouvement actuel d'échange disciplinaire entre les économistes et les sociologues pour repenser le champ de l'emploi et des relations professionnelles, dépassant les territoires traditionnels de la sociologie et de l'économie du travail. Dans la mesure où ce mouvement découle directement des transformations profondes qui affectent le marché du travail, et plus largement les modèles de relations construits autour de ces échanges économiques, Michel Lallement propose d'aborder la question de l'emploi comme l'élément structurant des rapports sociaux, ou comme il l'écrit lui-même, « comme une forme d'échange aux dimensions économiques, sociales et politiques » (p. 17).

Le premier chapitre de l'ouvrage développe les éléments conceptuels de l'approche privilégiée par l'auteur ainsi que la perspective dans laquelle il se situe. D'emblée, il choisit d'utiliser les catégories habituelles des relations professionnelles dans le cadre d'une approche institutionnaliste qui a « l'intérêt d'articuler les questions de relations professionnelles et d'emploi sur la base des conflits et des négociations à propos des règles et des frontières d'application de ces dernières », trouvant cette perspective plus fructueuse que la récente actualisation de l'interrogation des niveaux de négociation. Après avoir démontré les limites de cette dernière perspective,
Lallement propose d'actualiser 1'axiomatique institutionnaliste en centrant l'analyse sur l'enjeu de la fixation des frontières de l'espace conventionnel, c'est-à-dire en tant que communauté économique à l'intérieur de laquelle les acteurs interagissent au cours de processus associant production d'intérêt collectif et production de normes. Pour rendre compte de ces phénomènes, et en particulier pour permettre une démarche comparative, il propose trois paramètres d'analyse dans « un triple rapport [...] déterminant : rapport à l'État, rapports sociaux entre employeurs et représentants des intérêts salariés et, enfin, rapports économiques » (p. 42).

Dans le chapitre suivant, il procède à une problématisation de la réflexion précédente en abordant le cas particulier de la France. Suivant l'hypothèse que la France aurait progressivement abandonné le modèle dominant du « gouvernement de l'emploi » au profit d'une forme nouvelle de «gouvernance de l'emploi », le chapitre comprend deux parties: d'une part, une démarche de définition du champ des relations professionnelles dans le cadre du gouvernement de l'emploi caractérisé par la centralisation de la régulation étatique, par une structuration des relations professionnelles et par le rôle prépondérant de la grande entreprise hiérarchique. D'autre part, la caractérisation d'une gouvernance de l'emploi en émergence, dont les paramètres signalent une profonde transformation: la décentralisation vers le local, qui devient la cible des nouvelles politiques publiques de l'emploi, un mouvement d'autoréglementation des entreprises (les Américains parlent plutôt de corporate governance), enfin une nouvelle donne économique où se conjuguent le développement conjoint des entreprises supranationales et le développement de réseaux de petites entreprises. Les dernières sections du chapitre cherchent à souligner toute l'ambivalence de cette gouvernance en émergence. 
Les deux chapitres qui suivent visent à caractériser, avec plus de finesse nous dit l'auteur, quelques formes typiques de gouvernance de l'emploi. Comme de plus en plus d'analyses comparatives le suggèrent, Lallement aborde dans le premier le secteur d'activité économique comme un des espaces pour lequel cette perspective est particulièrement pertinente. Il y dessine les configurations particulières de trois secteurs bien différenciés : celui de la santé, le secteur du commerce de détail alimentaire, ainsi que le grand secteur de l'électronique, définissant pour chacun d'eux l'articulation spécifique entre les trois paramètres mentionnés plus haut. L'autre chapitre aborde la question du temps de travail et vise à présenter les nouvelles modalités de la flexibilisation du temps de travail de manière à rendre compte des pratiques locales de négociation. Comme dans les chapitres précédents, l'analyse est centrée sur les mutations de l'action publique, de l'évolution des relations entre les acteurs sociaux et de la dynamique de l'organisation économique.

Les deux chapitres de la dernière partie de l'ouvrage se tournent vers le cas allemand. Dans un premier temps, l'auteur cherche à démontrer les modalités spécifiques de la construction institutionnelle des relations professionnelles en Allemagne depuis la fin de la guerre. Selon lui, bien plus qu'en France, l'emploi y aurait été l'enjeu d'un échange de type politique. Dans ce chapitre, l'auteur s'appuie grandement sur les travaux du programme de recherche néocorporatiste. Il explique ainsi que, pour des raisons différentes selon les acteurs (le patronat craignait la « dérive inflationniste » alors que les syndicats voulait éviter "l'égoïsme d'entreprise $»$ ), on assiste graduellement à la construction de dispositifs néocorporatistes qui servent de médiation institutionnelle entre ces derniers. Mais, selon l'auteur, le grand virage de la réunification allemande viendra accélérer un mouvement de décentralisation des relations professionnelles qui étaient déjà perceptibles avant 1989 , remettant en cause les dispositifs macro-sociaux des arrangements institutionnels néocorporatistes. En particulier, la différenciation marquée entre les deux Allemagne encourage une plus grande diversité dans la stratégie des acteurs et ainsi une désarticulation des institutions traditionnelles gouvernant l'emploi. Dans l'autre chapitre, Lallement reprend pour l'Allemagne les divers thèmes abordés dans les chapitres précédents.

De manière générale, la réflexion menée par Michel Lallement est intéressante. En voulant s'inscrire dans le nouveau paradigme institutionnaliste en émergence, dans lequel il intègre le concept de gouvernance, il fait un choix pertinent. Les périodes de grandes transformations doivent en effet s'accompagner aussi d'une révolution paradigmatique. Or l'auteur semble avoir un problème à adopter les fondements véritablement originaux du nouveau paradigme. Par exemple, comme de nombreux autres sociologues ou politologues, Michel Lallement utilise le concept de gouvernance dans le vieux cadre de la pensée duale qui jadis opposait État/marché, qu'il ramène maintenant à celui de gouvernement/gouvernance. Il reconnaît bien la diversité des situations et la nouvelle pluralité des logiques d'action des acteurs, mais l'analyse se réduit bien souvent à opposer deux idéaux types irréductibles. Cette mauvaise utilisation du concept de gouvernance de l'emploi, qui ratisse excessivement large en englobant indistinctement tout ce qui n'est pas le «gouvernement de l'emploi ", ne peut déboucher que sur des conclusions vagues et prévisibles. L'auteur y échappe en proposant plutôt, en conclusion, de nouveaux axes de réflexions pour l'avenir.

Gilles L. BoURQUE Institut de recherche sur les PME Université du Québec à Trois-Rivières 